\title{
Inhalt/Contents 9/01
}

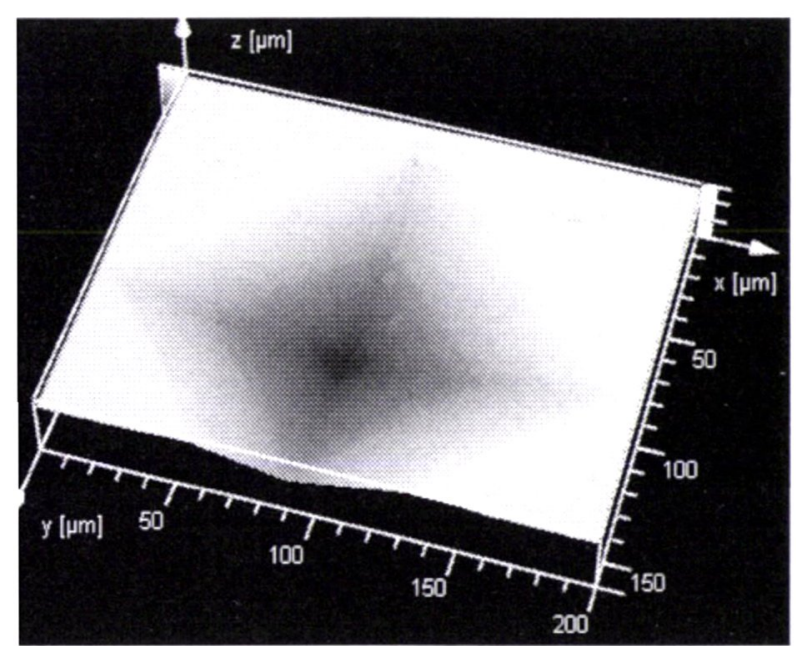

\section{Pohl}

Topologische Quantifizierung von Bauteiloberflächen mit dem CLSM

Topological Quantification of Component Surfaces by Means of CLSM

\section{J. Gegner, A. Öchsner \\ Digitale Bildanalyse in der quantitativen Metallographie Digital Image Analysis in Quantitative Metallography}

\section{A. Kulmburg, G. Kvas, G. Wiedner, P. Golob}

Gefügeaufbau von Co-Cr-Mo-(Nb)-Dentallegierungen

The Microstructure of Co-Cr-Mo-(Nb) Dental Alloys

Schadensanalyse / Failure Analysis

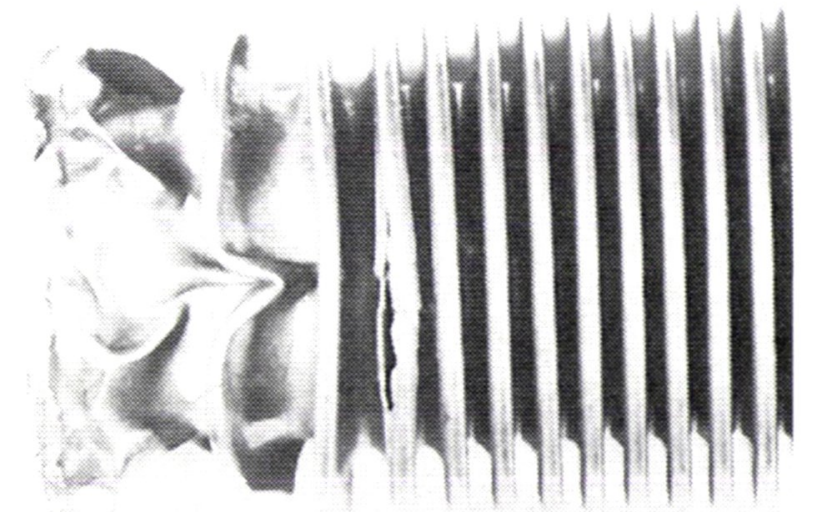

\section{H. Shaikh, G. George, H. S. Khatak}

Failure Analysis of Stainless Steel Bellows

- Mitteilungen/Information

- Literatur-Notizen/Literature Reviews 


\section{Praktische Metallographie}

\section{Practical \\ Metallography}

Gründer/Founders

Günter Petzow

Gerhard Reinacher +

Charlotte Wachau +

Herausgeber/Editor

Prof. Dr. G. Petzow

Schriftleiter

Editor in charge

Prof. Dr.-Ing. F. Mücklich

Universität des Saarlandes

Lehrstuhl für Funktionswerkstoffe

Postfach 151150

D-66041 Saarbrücken

Telefon: +49/681 302-2048

Telefax: +49/681 302-4876

E-Mail: pm-editor@matsci.uni-sb.de

Redaktion / Editor

Dipl.-Ing. S. Mücklich

Zöblitzer Str. 10

D-09125 Chemnitz

Telefon: $+49 / 371 / 531-5384$

Telefax: +49/371/531-6179

E-Mail: silke.muecklich@mbv.tu-

chemnitz.de

Dr. C. Bagnall, MCS Associates Inc., Greensburg, PA (USA

Dr. E. Bischoff, MPI für Metallforschung, Stuttgart

C. Bochert, Wirtz-Buehler GmbH, Düsseldorf

Prof. Dr. H.-E. Bühler, RWTH Aachen

V. Dietl, Lette Verein Berlin

Dr. G. Elssner, MPI für Metallforschung, Stuttgart

Prof. Dr. H.E. Exner, TU Darmstadt

Prof. Dr. E. Hornbogen, Ruhr-Universität Bochum

Prof. Dr. F. Jeglitsch, Montanuniversität Leoben

Dr. H.-J. Klaar, RWTH Aachen

Prof. Dr. A. KneissI, Montanuniversität Leoben

Dr. W.-U. Kopp, Daisendorf

Dr. J. Paul, Leica Vertrieb GmbH, Bensheim

Prof. Dr. M. Pohl, Ruhr-Universität Bochum

Prof. Dr. G. Schneider, Fachhochschule Aalen

U. Täffner, MPI für Metallforschung, Stuttgart

Dr. J. Trempler, Martin-Luther-Univ. Halle-Wittenberg

Prof. Dr. H.-H. Uchida, Tokai Univ., Hiratsuka, Kanagaw:

G. Vander Voort, Buehler Ltd., Illinois

Carl Hanser Verlag, München

HANSER

Prof. Dr. H. Vehoff, Univ. des Saarlandes, Saarbrücken

Dr. H.-J. Wieland, Verein Dt. Eisenhüttenleute, Düsseldor

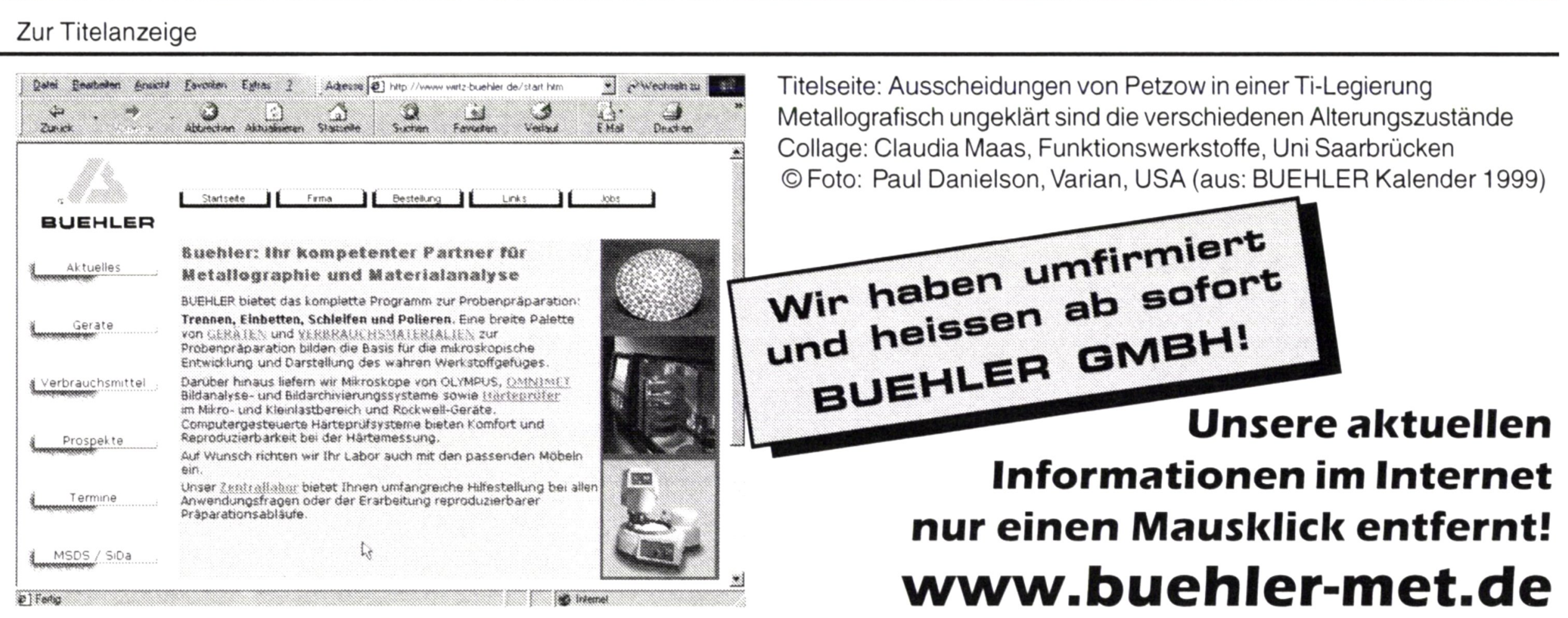

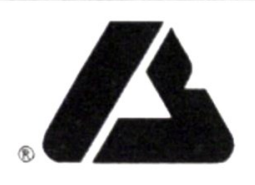

BUEHLER
BUEHLER GMBH • In der Steele 2 • D-40599 Düsseldorf • Tel. 0211/974100 • Fax 0211/9741079

BUEHLER GMBH • Postfach 515 • A-8700 Leoben • Tel. 03842/46676 • Fax 03842/46678

Prüfmaschinen AG • Postfach + CH-8953 Dietikon 1 • Tel. 01/7464030 • Fax 01/7464039

e-mail: vertrieb@ wirtz-buehler.de • http://www.buehler-met.de • http://www.buehler.com 